\title{
Roads as Channels of Centrifugal Policy Transfer: A Spatial Interaction Model Revised
}

\author{
Katarzyna Kopczewska'
}

ABSTRACT

\begin{abstract}
This paper proposes a methodology for measuring the spatial effects of roads and the seats of local authorities on the diffusion of business activity, which usually follows distance decay patterns from core to periphery. Regional development policies, pursued by regional authorities, directed at local units and designed to support local economies, are implemented by means of a centrifugal diffusion process. This invisible flow of policy is modeled using a one-way spatial interaction model represented by a multinomial distance decay function for the integrated spatial dataset. The research results indicate that NUTS5 (Nomenclature of Territorial Units for Statistics) units (gminas) perform better in terms of saturation with business activity when NUTS4 seats of authority are established there than when they are established near international roads. The natural diffusion process from core cities to the periphery covers approximately $25-30 \mathrm{~km}$, and the presence of international roads extends this range by $20 \mathrm{~km}$. The results confirm the hypothesis of an endogenous growth pattern.
\end{abstract}

KEY WORDS: $\quad$ spatial spillover; policy transfer; range of local governments; distance decay function

JEL Classification: $\quad H 7, \mathrm{H} 54, \mathrm{C} 21$

1 University of Warsaw, Poland

\section{Introduction}

Spatial characteristics are important to policy transfer. Regional and local governments interact in setting and pursuing policy. There are many spatial models of development, from core-periphery to polycentricity, that differ in how they describe the spatial distribution of social and economic activity. Depending on the spatial model of development considered, core localizations have different roles in creating the stimuli for spatial processes. The more centralized a region is, the stronger are the centrifugal stimuli needed to evoke spatial diffusion.

Correspondence concerning this article should be addressed to: Katarzyna Kopczewska, University of Warsaw Krakowskie Przedmieście 26/28, 00-927 Warsaw, Poland, e-mail: kkopczewska@wne.uw.edu.pl
Unequal forces over a whole administrative territory lead to geographic concentrations of business in space. Centrally located core territories attract the major of economic activity. Natural centrifugal diffusion resulting from agglomeration effects, such as searching for cheaper offices, avoiding over-congested roads, etc., strengthen the urban sprawl process. However, this applies only to suburban areas and first-row neighbors of cities. Interactions that reach further usually need some institutional support, which means that diffusion becomes a forced process. There is empirical evidence that the impact of core cities on surrounding areas (rural or nonmetropolitan) ranges no more than 25 miles (approximately $40 \mathrm{~km}$ ), including highways (Briggs, 1980; Lichter \& Fuguitt, 1980).

Business initiatives are usually attracted by public-sector activities that are implementations of 
development policies. Local interactions between businesses and the public sector are targeted to operate within the administrative boundaries of a regional territory. The extension of policies beyond regional boundaries would be inefficient in terms of regional costs and benefits, as a portion of the benefits would be consumed outside the region while all the costs would be incurred in the region. This means that local authorities (NUTS4 or NUTS5) cooperate mainly with their NUTS3 or NUTS2 authorities to contain policy effects inside the region (NUTS, Nomenclature of Territorial Units for Statistics). This sets the direction of policy flows from core to periphery and contain them inside the region.

Distance is a crucial issue here. According to Waldo Tobler's law (1970), "everything is related to everything else, but near things are more related than distant things." Local authorities located far from their regional authorities are likely to be less efficient in policy implementation than local authorities located closer. The first reason for this is the perception of policy as tacit knowledge that can only flow completely over short distances. Policy transfer can be perceived as a flow of tacit knowledge between agents that is dependent on distance-not only geographic distance but also cultural and social distances. Increasing spatial separation between local and regional authorities weakens policy flow and reduces the interactions between the core and the periphery (Dicken, 2007). Spatial concentration is needed for cooperation in the development of new approaches, the construction of social networks, and so forth. (Lundvall \& Johnson, 1994). The second reason is the spatial accessibility of territory, which permits easier flows to local areas that are well connected to the core city. Accessibility, understood as the ease with which a destination point can be reached from a given location using a certain transport system, can determine the economic potential of regions. According to Keeble et al. (1988), low-potential regions in the European Union generate low incomes. Standard spatial accessibility, in terms of territorial cohesion, etc., is often defined as a travel distance by road that does not exceed 60-90 minutes (Cinnamon et al., 2008; European Spatial Planning Observation Network, 2007) for access to palliative care or transportation to an airport. Unfortunately, there is little empirical evidence con- cerning the relation between spatial accessibility (or road networks or infrastructure investment) and the economic performance of regions and their business activity (Beuthe, 2002; Gutierrez et al., 2010).

However, distance and accessibility effects might be influenced by institutional effects. Having a seat of local self-government, which usually automatically converts a location into a local center, might be a business attraction factor. Many studies (e.g., Houghwout, 1999; Schaltegger \& Zemp, 2003) on city-suburban relations have shown that the decisions of authorities of neighboring communities are not independent from each other.

This leads to the main hypothesis that territories located on peripheries and not connected by highspeed roads may experience weaker policy implementation because of their lower attractiveness. Road access might facilitate the diffusion of business impulses. This diffusion would be a natural process, emerging when agglomeration diseconomies dominate. However, accessibility effects might be balanced by institutional factors. The seats of authorities, regardless of road connections, can attract greater business concentration than would result from the location factor only. An active public-sector policy can evoke driven diffusion when business opportunities are perceived. The first question is whether roads are significant channels in the acceleration of this diffusion process. The second question concerns the role of the seats of local authorities in attracting business activity. The overall question concerns the accumulation of business stimuli over space.

\section{Spatial integrated dataset}

Integrated spatial data permits economic analysis of spatial processes. Five types of data were merged for the purpose of the analysis described in this paper: data on the administrative divisions in Poland, data on the locations of county authorities, data on the road network, distance data and data on business indicators. All of the data were collected on the NUTS5 level. This level of aggregation minimizes the risk that some spatial trends might be hidden and ensures that edge effects will be as small as possible in spatial modeling.

Administrative division of the country - according to the NUTS classification adopted in European Union (EU) statistics, all NUTS5 regions belong to 


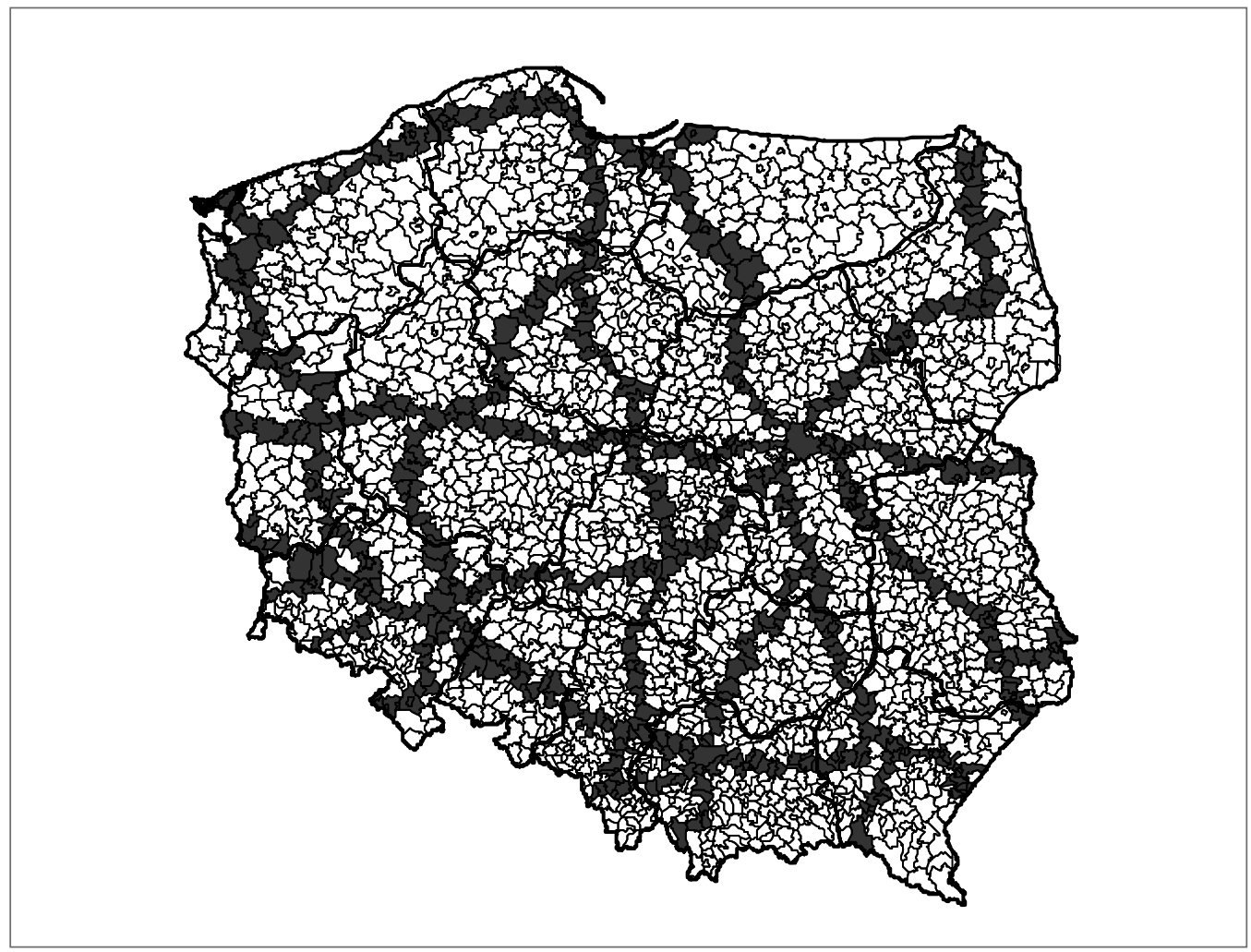

Figure 1. International roads in Poland in NUTS5 municipalities

higher NUTS levels. There are 2,478 NUTS5 municipalities (gmina), 379 NUTS4 counties (powiat) and 16 NUTS2 regions (województwo) in Poland. In all of these administrative units, local and regional governments have their seats. Regional development policy is designed on the NUTS2 level and then implemented on the NUTS5 and NUTS4 levels. NUTS5 and NUTS4 units are also responsible for undertaking local actions to support socio-economic development and growth. An average NUTS5 municipality has an area of 126 $\mathrm{km}^{2}$ and 15.5 thousand inhabitants, while a NUTS4 county has an average of 100 thousand inhabitants in an area of $825 \mathrm{~km}^{2}$. The territorial structure of the country must be taken into consideration in analyses concerning the public sector. Institutional influence is limited by territorial division and the structure of territories belonging to higher-order regions.

Institutional rent of NUTS4 powiat cities - intermediary government on the NUTS4 level was designed to carry out routine activities on the supra-municipal level. Labour market institutions are also located on this level. Powiat cities play roles as local centers, with local authorities, hospitals, secondary schools, geodesy specialists, etc. Municipalities that have the status of being local core cities are more important than other similar cities, mainly because they are closer to authorities and local decision centers. This level existed in Poland prior to 1975 and was re-introduced in 1999.

Roads - existing international public roads were taken into account in the analysis and included as a dummy variable on the NUTS5 level (Fig. 1). These roads are accessible from all of the municipalities through which they run. Only corridor express roads and highways with access limited to road junctions were excluded, as there is evidence that their impact might be negative (Rephann \& Isseman, 1994). The total length of the main national public roads in Poland is $18,368 \mathrm{~km}$, of which $5,500 \mathrm{~km}$ are international 


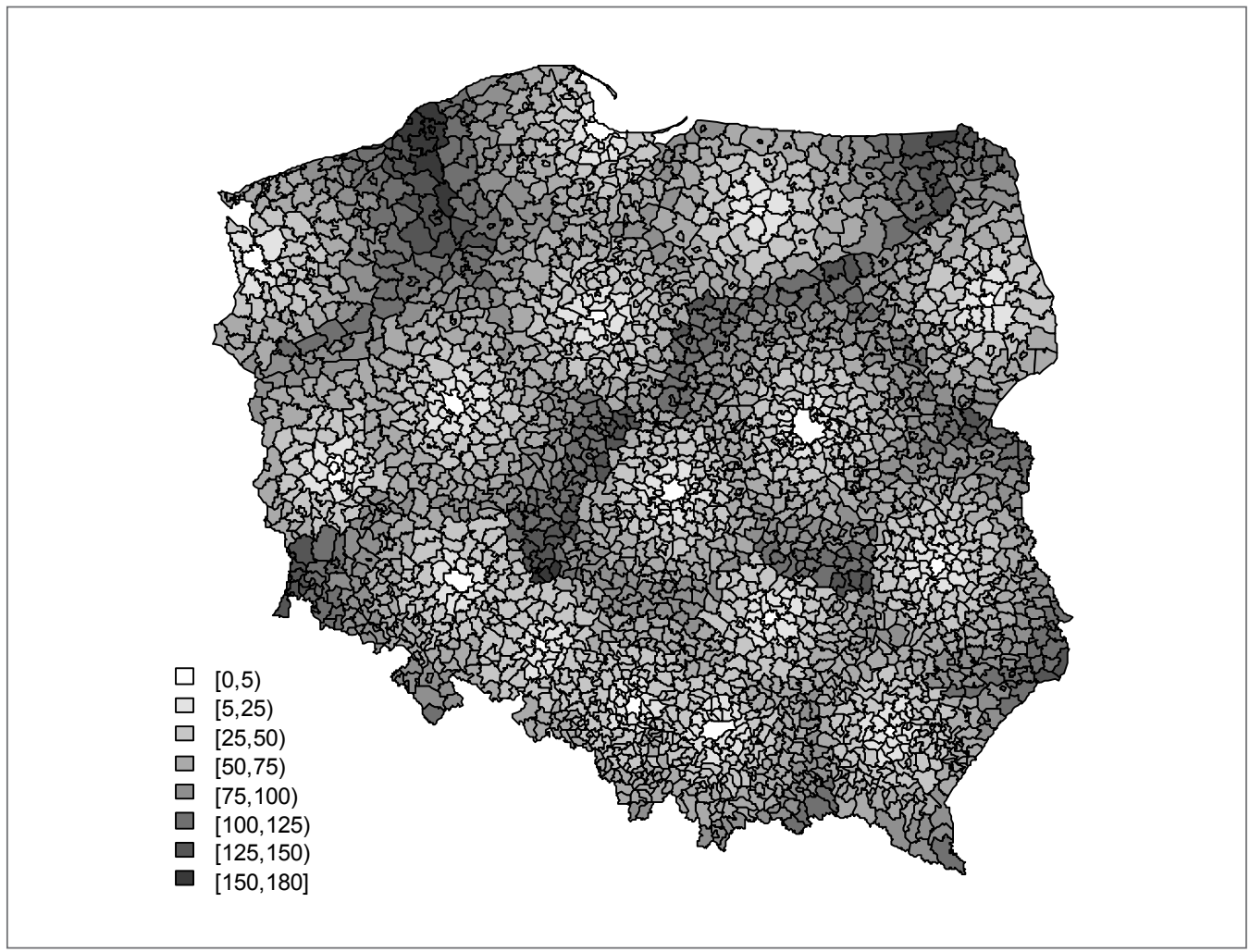

Figure 2. Euclidean distance between NUTS5 municipalities and their regional core cities

public roads, $916 \mathrm{~km}$ are highways, $364 \mathrm{~km}$ are express dual-lane roads and $242 \mathrm{~km}$ are single-lane roads. Main national roads make up approximately $5 \%$ of all the public roads in the country-an area of $7,730 \mathrm{~km}^{2}$ of a total area of $312,679 \mathrm{~km}^{2}$ of roads in Poland. The unequal spatial distribution of the roads has its roots in the historical division of the country. The density of roads (the proportion of the area covered by roadway infrastructure) measured in NUTS2 regions ranges from $4 \%$ in the eastern and northern parts of the country to more than $8 \%$ in the southern part. International roads are located in approximately $22 \%$ of the country's municipalities.

Distance - the Euclidean distances between NUTS5 territories and their respective main regional cities were calculated as a measure of spatial separation. Using this approach, naturally, the problems of natural barriers, road networks, travel times, etc. are encountered. For this reason, more sensitive studies use sophisticated measures of distance, such as road distances in $\mathrm{km}$, travel times and travel costs, applying optimal route calculations. However, reliable information of this type is not commonly available for local units.

NUTS5 municipalities cooperate with the corresponding NUTS2 regional authorities in providing goods and services and implementing social, economic and environmental policies. After the territorialadministrative reform of 1999, when 49 regions were reduced to 16 NUTS2 units, there were municipalities that were located approximately $180 \mathrm{~km}$ from a core city. A large distance from a core city usually means worse accessibility (Fig. 2). Travel time can be approximated based on Euclidean distance. For the Polish road network, one can assume that a 1-km Euclidean distance $=$ a $1.2-\mathrm{km}$ road distance and that a $1-\mathrm{km}$ road distance $=1.06$ minutes of travel time. For a random sample of 100 municipalities within 50, 100 and 150 $\mathrm{km}$ from a central city, road distances and estimated 


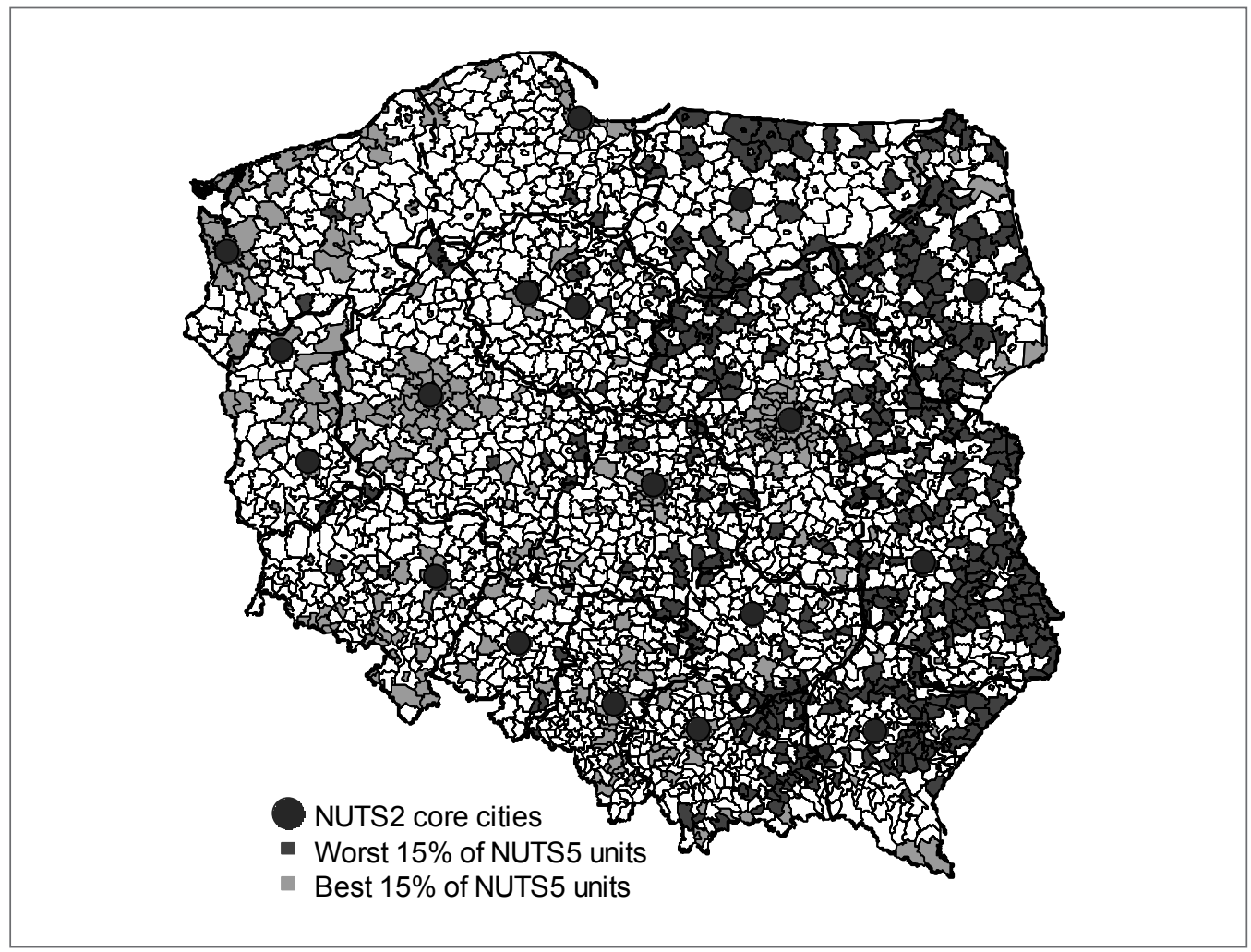

Figure 3. Spatial distribution of business activity per capita (2009)

travel times were calculated with the use of a web-map. The results are regression coefficients with significance levels less than 0.00001. Similar multipliers were obtained by Tobler (1993).

Business indicators - for every NUTS5 municipality, the number of firms per 1,000 inhabitants was calculated for the year 2009. In the Polish economy, there are 3,74 million business entities, 95\% of which are small companies (with less than $10 \mathrm{em}-$ ployees) and approximately $4,3 \%$ of which are average-size business units (with less than 50 employees). The spatial distribution of these companies is nonuniform (see Fig. 3) -ranging from 65 units per 1,000 people in PL09 Podkarpackie (in southeastern Poland) to 122 units per 1,000 people in PL0G Zachodniopomorskie (in northwestern Poland). On the regional level, business stimuli and attitudes are the consequences of historical circumstances, culture, development level, endogenous resources, etc. On the local level, when all those factors are uniform within a region, location and institutions matter. Market forces tend to locate business units in the most attractive places: local centers and/or the most accessible locations.

\section{One-way spatial interaction model}

The flows of goods and services between two destinations, which decrease with distance, are usually analyzed using spatial interaction models, which have been widely applied in studies of transportation, migration, trade, policy diffusion, research impact, knowledge flows, innovation implementation, etc. (Taylor, 1975; Vries, Nijkamp \& Rietveld, 2005). The basic model of spatial interactions assumes the existence of a $T$ matrix of $T_{i j}$ flows between locations from origin $\mathrm{M}$ to destination $\mathrm{N}(1)$, a $d$ matrix of the $d_{i j}$ distances between locations (2), and a $T^{\prime}$ matrix of $T^{\prime}{ }_{i j}$ theoretical values of flows (3). 
$T=\left\{T_{i j}\right\}_{i, j=1}^{M . N}$

$d=\left\{d_{i j}\right\}_{i, j=1}^{M . N}$

$T^{\prime}=\left\{T_{i j}^{\prime}\right\}_{i, j=1}^{M, N}$

In spatial interaction models, flows are assumed to be functions of distance (or of other spatial separation measures, so-called transport friction) and of the characteristics of the origin and destination locations (control variables). A set of variables that control the flows is complemented by other explanatory variables-very often, population, employment or income variables. To avoid multicollinearity in cases in which the dependent variable is in per capita terms, the set of attributes considered should include other information, such as socio-political patterns, institutional attributes of the locations, etc.

The assumption of paired two-way flows is important in business models of trade, migration, etc. In the case of policy transfer, with flows of development and innovation incentives or tacit knowledge, which are usually not observed, it is required to assume one-way flows from the core to the periphery. This is consistent with institutional settings in which core regional authorities set up roadmaps of activities for local authorities in peripheral areas. Assuming that one core sends an impulse to many peripheries and that the flows are centrifugal, the $d$ matrix and $T$ matrix became a vector. In models of this type, the flows of policy are invisible; only their results can be measured. Unequal spatial distribution in the process examined is explained by localization, defined in terms of the distance to a core city, high accessibility (e.g., by highspeed roads) and the characteristics of the neighbors.

As distance decay models describe flows with respect to distance, there are various possible functions, such as exponential (4), power (5) and polynomial (6) functions, that can be applied:

$\ln x_{1}=\beta_{0}+\beta_{1} D+e$

$\ln x_{1}=\beta_{0}+\beta_{1} \ln D+e$

$x_{1}=\beta_{0}+\beta_{1} x_{0}+\phi_{1} D^{1}+\phi_{2} D^{2}+\phi_{3} D^{3}+\phi_{4} D^{4}+\ldots+e$

where $x_{1}$ is the level of a proxy that indicates the result of policy flows and D is the distance between the core and the examined territory.
The function selection problem has been widely discussed in the literature. Fotheringham and O'Kelly (1989) have proposed classifications to reflect the relevance of a function to the research problem, as well as advanced models of two-way flow, such as that described by LeSage and Pace (2009). There is also a long list of advantages and disadvantages to be considered in function selection. For example, a power function is better than an exponential function when it is necessary to ensure comparability of parameters between the tests, regardless of the measurement scale (Fotheringham \& O'Kelly, 1989). In a detailed discussion of spatial filters for two-way models, Griffith (2007) explained that spatial autocorrelation and distance decay intermingle in spatial interaction model specifications. However, this discussion usually does not address polynomial functions, which may change the direction of interaction in the $+\infty$ zone, or their ability to predict negative interactions when the function falls below the $x$ axis, or ambiguous interpretation of the constant term (Taylor, 1975). On the other hand, polynomial functions are more flexible in fitting to the data. Polynomial models are often used in trend surface analysis (Legendre \& Legendre, 1998).

Several measures can be used for model quality assessment. The basic $\mathrm{R}^{2}$ parameter is recommended for use in OLS calibration, the Information Gain I parameter is recommended for use in the MLE calibration, and finally, the SRMSE (Standarized Root Mean Square Error) is useful regardless of the functional form and estimation method.

SRMSE $=\frac{\sqrt{\frac{\sum_{i, j=1}^{M, N}\left(T_{i j}-T_{i j}^{\prime}\right)^{2}}{M \cdot N}}}{\frac{\sum_{i . j=1}^{M, N} T_{i j}}{M \cdot N}}$

The interpretation of SRMSE employs a rule of thumb with the following thresholds: SRMSE values between 0 and 0.5 mean a very good fit, SRMSE values up to 0.75 represents moderate ability to reflect major trends only, and SRMSE values between 1 and $\infty$ mean a poor fit, often with off-scale observations (Andersson et al., 2008).

Spatial interaction models, irrespective of the functional form, are usually estimated using classical aspatial methods. Recently, two approaches have 


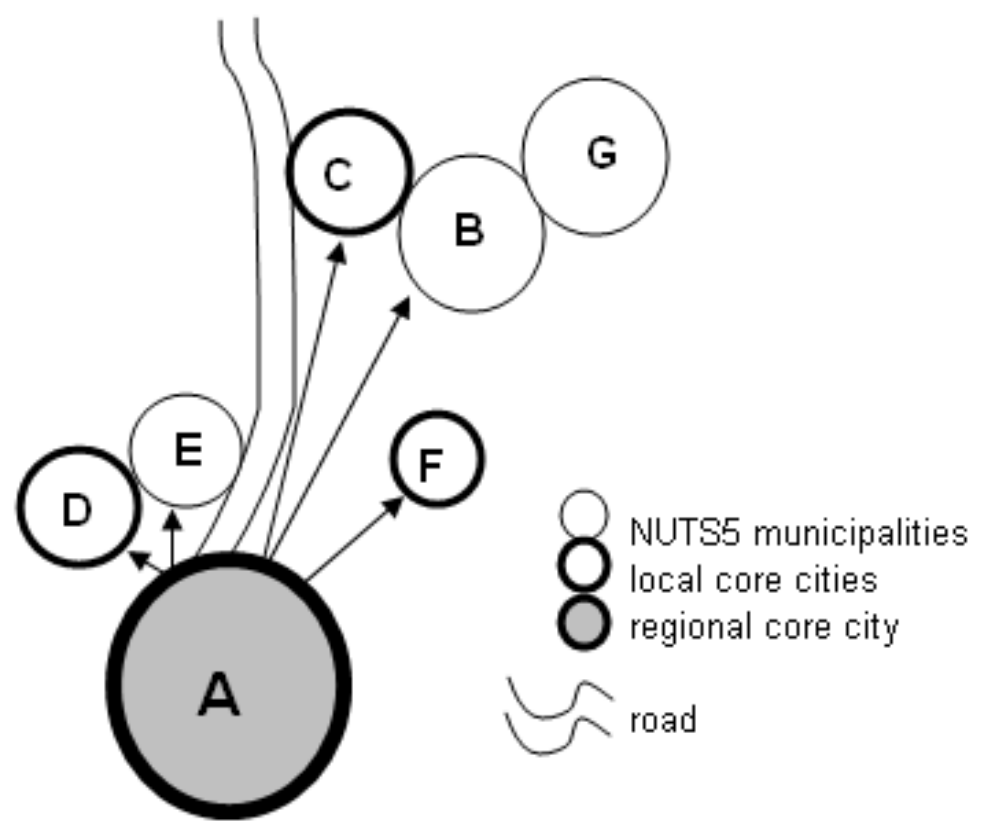

Figure 4. Spatial interactions approach

been presented in the literature that use eigenfunction-based spatial filtering or spatial econometrics to address spatial autocorrelation in the residuals (Fisher \& Griffith, 2006). In addition, for one-way invisible flows, the spatial structure and neighborhood matrix should be included in the estimation, which controls the spatial autocorrelation of the residuals. Assuming the relation illustrated in Fig. 4, where B, C and G are peripheral neighbors, and $A$ is the distant core, spatial autocorrelation may exist in the $\mathrm{A}-\mathrm{B}, \mathrm{A}-\mathrm{C}$ and $\mathrm{A}-\mathrm{G}$ pairs because of the similarity of the interactions. In addition, the diffusion of development stimuli from $\mathrm{A}$ to $\mathrm{D}$ and $\mathrm{E}$ will be stronger than the diffusion to $\mathrm{C}, \mathrm{B}$ and $\mathrm{G}$. Better accessibility of $\mathrm{E}$ and $\mathrm{C}$ than of D, B and G may also strengthen the invisible flow. However, stronger flow of intangible assets should be evident in the proxy data for the regions.

Identification of a proxy for invisible flow requires good institutional orientation with respect to the possible connections. If business development stimuli are analyzed, a possible proxy would be the number of companies per capita (or to the working population) in a given region. The ratio of the number of insolvent and bankrupt companies to the number of newly established firms might shed light on the policy transmission process.

A spatial structure can be incorporated in the model using a spatial weights matrix. In the case of spatial one-way flow models, an inverse distance matrix will duplicate information about the covariates of the distance. The contiguity matrix, in which only common borders matter, fits the problem theoretically, as the local flows occur with the closest neighbors. Also a matrix of higher than the first row order can be applied. Ex post results will confirm choices made a priori. The structural form of a spatial model-i.e., whether it models spatial lag or spatial error-depends on spatial process characteristics and can be tested with LM models on the basis of OLS residuals. Spatial estimation details can be found in Cohen (2010). 
Table 1. Empirical statistics for business units per 1000 inhabitants depending on distance ( $\mathrm{km})$, road network and institutional settings

\begin{tabular}{|c|c|c|c|c|c|c|c|}
\hline & $0 \mathrm{~km}$ & $<25 \mathrm{~km}$ & $25-50 \mathrm{~km}$ & $50-75$ km & $75-100 \mathrm{~km}$ & $100-125 \mathrm{~km}$ & $125-150 \mathrm{~km}$ \\
\hline General average & 136 & 88.5 & 68.2 & 65.7 & 68.1 & 66.66 & 70.7 \\
\hline $\begin{array}{l}\text { No roads, no authorities' } \\
\text { seat }\end{array}$ & NA & 83.2 & 60.7 & 59.5 & 62.3 & 59.1 & 62.1 \\
\hline $\begin{array}{l}\text { Roads included, no } \\
\text { authorities'seat }\end{array}$ & NA & 90.4 & 69.9 & 70.6 & 67.2 & 59.9 & 72.5 \\
\hline $\begin{array}{l}\text { No roads, authorities'seat } \\
\text { included }\end{array}$ & 130,1 & 109.3 & 96.3 & 98.2 & 97.7 & 106.7 & 109.3 \\
\hline $\begin{array}{l}\text { Both roads and } \\
\text { authorities'seat included }\end{array}$ & 137 & 100 & 105.2 & 108.4 & 115.1 & 107.9 & 134.0 \\
\hline
\end{tabular}

\section{Estimation results}

A model for policy diffusion was estimated using Polish NUTS5 data using the R software's $s p$ and $s p d e p$ packages (Bivand et al., 2010; R Core Team, 2010). Local development of business (in per capita terms) was found to be strongly dependent on distance but also on road network characteristics and institutional characteristics (control variables):

$$
B=f(\text { Dist, } \text { Attributes })
$$

where B are the flows of business in the per capita approach, the core factor Dist is the Euclidean distance between units, and attributes represents control variables such as road network characteristics and institutional characteristics.

Spatial effects are clearly evident, both in the empirical statistics (Table 1) and the estimated theoretical values (Table 2). There is a significant effect of business spatial concentration in local core cities. Municipalities located on peripheries (far from core cities with poor accessibility because of lack of access to international roads) without local authorities have, on average, less than half of the business units located in regional core cities. The empirical data indicate that when a road runs through a municipality, the number of business units per capita is higher by approximately $15 \%$ on average. An administrative decision to locate the seat of a local authority in a local city increases the number of business units by approximately $60 \%$. The combination of the two (roads and seats) improves municipality performance by approximately $75 \%$. The effect of local governments seems to be on four times stronger average than the effect of road, but the further an area is located from a core city, the stronger the spatial effects observed are.

Three model types were tested-multinomial, power and exponential models-and estimated as nonspatial and spatial models. The form of the spatial error model was chosen on the basis of LM tests on the OLS residuals. The first main issue in this analysis is the choice of a model. The standardized root mean square error (SRMSE $>1$ ) indicates that power and exponential model forms do not fit the data well. A fourth-degree polynomial model is much better than those other two options and fits the data well (SRMSE=0.3-0.4). In addition, spatial models, justified by the significant lambda in the regression and significant positive Moran I for OLS residuals are much better fitted than nonspatial ones (as indicated by better AIC and SRMSE values). Misspecification of nonspatial models results in bias, i.e., overestimation of parameters. A spatial error model filters out spatial autocorrelation caused by the similarity of flows to locations at equal distances.

The second important point is the meaning of the distance from the core city. When no spatial effects-roads or authorities seats-exist, approximately $25-30 \mathrm{~km}$ is a sufficient distance to characterize the core-periphery 
Table 2. Estimation results - number of business units per 1000 inhabitants as explained by distance, road network and NUTS4 authoroties' seats.

\begin{tabular}{|c|c|c|c|c|c|c|}
\hline \multirow{2}{*}{$\begin{array}{l}\text { Model } \\
\text { Covariates }\end{array}$} & \multicolumn{2}{|c|}{$\begin{array}{l}\text { Polynomial } \\
\qquad \begin{array}{l}y \sim(x)\end{array}\end{array}$} & \multicolumn{2}{|c|}{$\begin{array}{c}\text { Power } \\
\log (y) \sim f(x)\end{array}$} & \multicolumn{2}{|c|}{$\begin{array}{l}\text { Exponential } \\
\log (y) \sim f(x)\end{array}$} \\
\hline & a-spatial & spatial & a-spatial & spatial & a-spatial & spatial \\
\hline Constant & $103.5^{* * *}$ & $97.3^{* * *}$ & $4.47^{* * *}$ & $4.29 * * *$ & $4.16^{* * *}$ & $4.20^{* * *}$ \\
\hline Log(Dist) & -- & --- & $-0.10^{* * *}$ & $-0.04^{* *}$ & --- & -- \\
\hline Dist & $-2.16^{* * *}$ & $-1.50^{* * *}$ & --- & --- & $-0.0015^{* * *}$ & $-0.0013^{* * *}$ \\
\hline Dist2 & $3.8 \mathrm{e}-2^{* * *}$ & $2.3 e-2^{* * *}$ & --- & -- & --- & --- \\
\hline Dist3 & $-2.8 e-4^{* * *}$ & $-1.4 e-4^{*}$ & -- & -- & -- & -- \\
\hline Dist4 & $7.4 \mathrm{e}-7^{* * *}$ & $3.06 e-7^{*}$ & -- & -- & -- & --- \\
\hline Road & $7.7^{* * *}$ & $4.51^{* * *}$ & $0.12^{* * *}$ & $0.06^{* * *}$ & $0.13^{* * *}$ & $0.067^{* * *}$ \\
\hline Seat of auth. & $35.6^{* * *}$ & $34.0^{* * *}$ & $0.47^{* * *}$ & $0.44^{* * *}$ & $0.48^{* * *}$ & $0.45^{* * *}$ \\
\hline SRMSE & 0.387 & 0.303 & 1.04 & 1.04 & 1.04 & 1.04 \\
\hline $\mathrm{AIC}$ & 23356 & 22414 & 1623.6 & 403.31 & 1670 & 340 \\
\hline Moran's I & $0.44^{* * *}$ & -0.07 & $0.51^{* * *}$ & -0.07 & $0.52^{* * *}$ & -0.07 \\
\hline Lambda & -- & $0.68^{* * *}$ & --- & $0.73^{* * *}$ & -- & $0.73^{* * *}$ \\
\hline
\end{tabular}

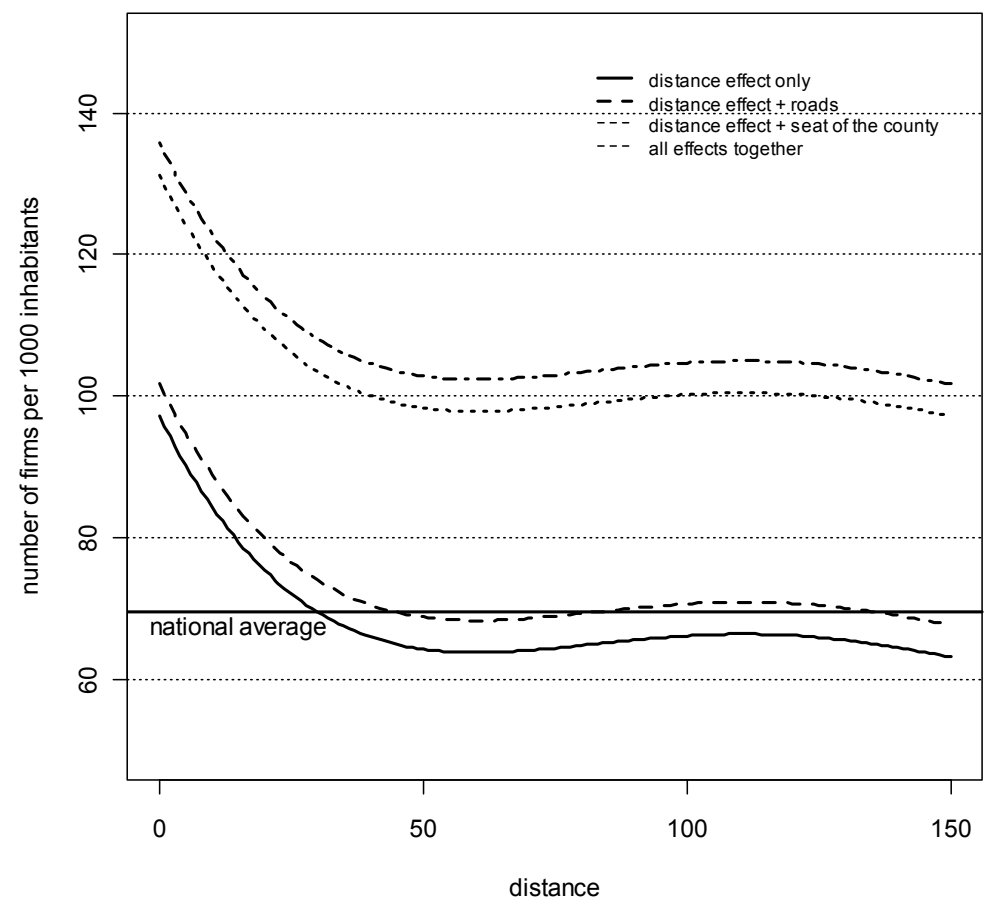

Figure 5. Fitted values of polynomial model 
diffusion process (Fig. 4). According to the fitted curve, the number of business units per 1,000 inhabitants falls below the national average. Most of the municipalities located further than $30 \mathrm{~km}$ from city centers are weaker than average in terms of business saturation.

The third point pertains to disturbances over the space. The natural spatial diffusion and cumulative effects of distance are changed by accessibility and institutional settings. International roads extend the range of the diffusion by approximately $20 \mathrm{~km}$ to approximately $45-50 \mathrm{~km}$. On average, the number of business units per 1,000 inhabitants is $5-7 \%$ higher then without access to a road. When a NUTS5 municipality is a seat of NUTS4 authorities (no international roads included), the number of business units is $35-55 \%$ higher then without the local authority seat. The status of being the local core ensures that business saturation almost never falls below 100 units per 1000 inhabitants, which is 30 units higher, on average, than without having local authorities in a city.

\section{Conclusions}

This study of spatial diffusion of business activity in the core-periphery pattern captured the effects of international roads and institutional settings on natural flow mechanisms. NUTS5 municipalities connected administratively with regional NUTS2 core cities exhibit different degrees of business saturation, which was hypothesized to decrease with distance. A oneway spatial interaction model, specified as a fourth-order polynomial function and estimated as a spatial error model, proved that distance is indeed a significant factor in the diffusion process. However, this might be influenced by accessibility and institutional effects.

The results indicate that economic development might be stimulated by expanding the road network to facilitate business flows from the core to the periphery. Better two-way accessibility, from central to local units and vice versa, increases business saturation by $5-7 \%$ and extends the core range by $20 \mathrm{~km}$. Much stronger spatial effects are achieved with institutional settings. NUTS4 authorities facilitate the self-government of NUTS5 authorities, which are responsible for local development. The duties of NUTS4 authorities are to work with NUTS5 units to provide public goods and services on the supra-local level. Their impact on the business environment is much greater than the influ- ence of roads, and one should expect a $35-55 \%$ increase in the number of business units per 1,000 inhabitants in other locations. Those results are consistent with existing empirical evidence of the weak impact of transportation infrastructure on regional production (Garcia-Mila \& McGuire, 1992; Munnell, 1990).

Those results indicate that the core-periphery spatial diffusion process is rather poor, although it was implemented in Polish regional policy at last 15 years ago. The economic development in local NUTS4 seats is more a matter of local interactions than flows from the core. This means that idea of implementation of endogenous development has a place here. This leads to conclusion that a spatial cohesion policy should be implemented at the local level. Local core cities and their accessibility are the most important elements in developing business environments.

\section{References}

Andersson, J., Joernsten, K., \& Pettersen Strandenes, S. (2008). Modeling Freight Markets for Coal. NHH (Discussion Paper December 26, 2008). INSTITUTT FOR FORETAKSØKONOMI.

Beuthe, M. (2002). Transport evaluation methods: from cost-benefit analysis to multicriteria analysis and the decision framework. In L. Giorgi \& A. D. Pearman (Eds.), Project and Policy evaluation in Transport (pp. 215-243). Aldershot, UK: Ashgate.

Bivand, R., Altman, M., Anselin, L., As-sunçao, R., Berke, O., Bernat, A., ... Yu, D. (2010). spdep: Spatial dependence: weighting schemes, statistics and models (R package version 0.5-21) [Software]. Available from: http://CRAN.R-project.org/package=spdep

Briggs, R. (1980). The impact of interstate highway system on non-metropolitan growth. Washington, DC: US Department of Transportation.

Cinnamon, J., Schuurman, N., \& Crooks, V. (2008). A method to determine spatial access to specialized palliative care services using GIS. BMC Health Services Research, 8 (140). doi:10.1186/1472-69638-140. Retrieved from: http://www.biomedcentral.com/1472-6963/8/140

Cohen, J. P. (2010). The broader effects of transportation infrastructure: Spatial econometrics and productivity approaches. Transportation Research Part E - Logistics and Transportation Review, 46 (3), 317-326. 
Dicken, P. (2007). Global Shift. Mapping the Changing Contours of the World Economy (5th ed.). London, UK: SAGE Publications Ltd.

European Spatial Planning Observation Network. (2007). Scenarios on the territorial future of $\mathrm{Eu}$ rope. ESPON Project 3.2 Final Report, Luxemburg. Retrieved from http://www.espon.eu/ export/sites/default/Documents/Publications/ ESPON2006Publications/SpatialScenarios/ espon3.2_60p._final_16-7-2007-c.pdf

Fisher, M. M. \& Griffith, D. A. (2006). Modeling Spatial Autocorrelation in Spatial Interaction Data: A Comparison of Spatial Econometric and Spatial Filtering Specifications (ERSA Conference Paper No. 06p10). European Regional Science Association.

Fotheringham, A. S, \& O’Kelly, M. E. (1989). Spatial Interaction Models: Formulations and Applications, London, UK: Kluwer Academic Publishing.

Garcia-Mila, T., \& McGuire, T. J. (1992). The contribution of publicly provided inputs to states' economies. Regional Science and Urban Economics, 22 (2), 229-242.

Gutierrez, J., Condeco-Melhorado, A., \& Martin, J. C. (2010). Using accessibility indicators and GIS to assess spatial spillovers of transport infrastructure investment. Journal of Transport Geography, 18 (1), 141-152.

Griffith, D. A. (2007). Spatial Structure and Spatial Interaction: 25 Years Later. The Review of Regional Studies, 37 (1), 28-38.

Houghwout, A. F. (1999). Regional Fiscal Cooperation in Metropolitan Areas: An Exploration. Journal of Policy Analysis and Management, 18 (4), 579-600.

Keeble, D., Offord, J., \& Walker, S. (1988). Peripheral regions in a community of twelve. Luxembourg: Office for Official Publications of the European Communities.

Legendre, P., \& Legendre, L. (1998). Numerical Ecology (2nd ed.). Amsterdam: Elsevier Science.

LeSage, J., \& Pace, R. K. (2009). Introduction to Spatial Econometrics. London, UK: CRC Press Taylor \& Francis Group.

Lichter, D. T., \& Fuguitt, G. V. (1980). Demographic response to transportation innovation: the case of the interstate highway. Social Forces, 59 (2), 492-511.

Lundvall, B. A., \& Johnson, B. (1994). The learning economy. Journal of Industry Studies, 1 (2), 23-42.
Munnell, A. H., Cook, L. M. (1990). How does public infrastructure affect regional economic performance. New England Economics, Sep, 11-33.

R Core Team (2010). R: A language and environment for statistical computing. $R$ Foundation for Statistical Computing [Software], Vienna, Austria. Available from: www.r-project.org

Rephann, T., \& Isserman, A. (1994). New highways as economic development tools: An evaluation using quasi - experimental matching methods. Regional Science and Urban Economics, 24 (6), 723-751

Schaltegger, C. A., \& Zemp, S. (2003). Spatial Spillovers in Metropolitan Areas: Evidence from Swiss Communes (Working Paper No. 2003-06). Center for Research in Economics, Management and the Arts.

Taylor, P. (1975). Distance Decay Models in Spatial Interactions. CATMOG (Concepts and Techniques in Modern Geography) No. 2. Retrieved from http:// qmrg.org.uk/files/2008/11/2-distance-decay-inspatial-interactions.pdf

Tobler, W. (1970). A computer movie simulating urban growth in the Detroit region. Economic Geography, 46 (2 Suppl: Proceedings. International Geographical Union. Commission on Quantitative Methods), 234-240.

Tobler, W. (1993). Speculations On The Geometry Of Geography (Technical Report No. 93-1). National Center For Geographic Information And Analysis. Retrieved from http://www.ncgia.ucsb.edu/ Publications/Tech_Reports/93/93-1.PDF

Vries, J., Nijkamp, P., \& Rietveld, P. (2005, August, 24). Exponential or power distance-decay for commuting? An alternative specification. Paper presented at 45th Congress of the European Regional Science Association, Amsterdam. 
